\title{
Optimizing a serum-free/xeno-free culture medium for culturing and promoting the proliferation of human dental pulp stem cells
}

\author{
Esraa Mohamed Abdel Moniem ${ }^{1,2}$, Mona Mahmoud EL-Batran ${ }^{1}$, Ahmed Mahmoud Halawa, \\ Dina Hazem Gomaa ${ }^{3}$, Ghada Nour Eldeen ${ }^{4}$, Riham Mohamed Aly ${ }^{1,2}$ \\ ${ }^{1}$ Department of Basic Dental Science, ${ }^{2}$ Stem Cell Laboratory, Center of Excellence for Advanced Sciences, National Research Centre, Cairo, Egypt; \\ ${ }^{3}$ Department of Oral Biology, Faculty of Dentistry, Ain Shams University, Cairo, Egypt; ${ }^{4}$ Department of Molecular Genetics and Enzymology, \\ National Research Centre, Cairo, Egypt \\ Contributions: (I) Conception and design: MM EL-Batran, AM Halawa, DH Gomaa, RM Aly; (II) Administrative support: None; (III) Provision \\ of study materials or patients: EM Abdel Moniem; (IV) Collection and assembly of data: EM Abdel Moniem, RM Aly; (V) Data analysis and \\ interpretation: EM Abdel Moniem, GN Eldeen, DH Gomaa, RM Aly; (VI) Manuscript writing: All authors; (VII) Final approval of manuscript: All \\ authors. \\ Correspondence to: Riham Mohamed Aly. Department of Basic Dental Science, Oral \& Dental Research Division, National Research Centre, 33 El \\ Buhouth St., Dokki, Cairo 12622, Egypt. Email: Riham.aly@hotmail.com.
}

Background: Dental pulp stem cells (DPSCs) hold great promise for utilization in tissue repair and regenerative medicine. Routinely, culture media used for culturing stem cells are supplemented with animal serum for promoting growth and successful maintenance of stem cells. However, there is a growing demand for optimizing a well-defined culture media that could safely increase the efficacy and reproducibility of the cultured cells. In this study, we aimed at optimizing a serum-free/xeno-free culture medium.

Methods: A cocktail of various supplements intended to enrich DPSCs proliferation in defined concentrations was designed. It consisted of recombinant human basic fibroblast growth factor (hbFGF), insulin transferrin selenium (ITS), ascorbic acid (vitamin C), Beta mercaptoethanol and cholesterol. The effect of this optimized media on the proliferation of DPSCs was assessed by MTT assay and flow cytometric analysis (FACS) of early apoptotic marker annexin V. Expression of stemness-related genes (OCT4, SOX and NANOG) was assessed by qRT-PCR.

Results: Proliferation results by MTT illustrated a significant increase in the proliferation rate of DPSCs cultured in the proposed media. FACS analysis of annexin V expression was nil. Expression of OCT4, SOX and NANOG genes was also up-regulated.

Conclusions: The proposed combination of supplements utilized in the proposed culture media successfully increased the proliferation potential of DPSCs in addition to enhancing the stemness properties. Thus, it can be considered a promising and safe substitute to traditional animal derived supplements like fetal bovine serum (FBS).

Keywords: Culture medium; dental pulp stem cells (DPSCs); serum free; xeno free

Received: 12 February 2019; Accepted: 15 May 2019; Published: 27 June 2019.

doi: $10.21037 /$ sci.2019.06.05

View this article at: http://dx.doi.org/10.21037/sci.2019.06.05

\section{Introduction}

Stem cells are unique cells that have the capability to undergo unlimited divisions giving rise to identical, undifferentiated cells. Using appropriate stimuli, these cells can be induced to differentiate into multiple cell types (1-3).
Previous researches have highlighted the role of the dental pulp tissue as a source of mesenchymal stem cells (MSCs) that can be differentiated into several cell types. The advantage of dental pulp stem cells (DPSCs) is that it involves a minimally invasive process of acquisition in 
addition to their long-term cryopreservation capacity. Examples of diseases that are currently being studied for potential cure by DPSCs include; type I diabetes, diseases of neurological origin, immunodeficiency diseases and bone diseases (4). Routinely, culture media in which stem cells are grown are supplemented by animal serum for promoting growth, successful maintenance and differentiation of stem cells. Fetal bovine serum (FBS) is now considered a global growth adjuvant effective for almost all cell types. FBS constitutes a combination of most of the factors essential for cell attachment and proliferation; nevertheless, disadvantages may arise either due to batchto-batch variations or due to the underlying risk of possible contamination with endotoxins, mycoplasma, and viral contaminants. Also, the controversy involving the methods of collection of FBS from bovine foetuses presents an ethical issue (5). There is currently an urge to decrease the dependence on animal derived sera, thus the present study aimed to develop a serum-free/xeno-free (SF/XF) culture medium that could safely enhance the proliferation of DPSCs.

\section{Methods}

\section{Cell isolation and culture}

All experiments conducted in this study have been reviewed and were granted approval by the Ethical Committee of the National Research Centre and Faculty of Dentistry (Ain Shams University). Teeth were collected from healthy patients undergoing extraction at the oral surgery clinics of the National Research Centre clinics. In this study, six healthy donors ( 3 males and 3 females) aged from 16 to 25 undergoing extraction of impacted wisdom molars were included. Donors were healthy and did not present any history of illness. Also, all teeth were sound and showed no signs of caries with healthy periodontium. Informed consents were obtained from donors prior to extraction. Chlorhexidine mouth rinse was administered before undergoing extraction to reduce oral microbial flora. DPSCs isolation followed previously published protocol (6). The extirpated pulp tissues were triple rinsed in PBS, and were cut into tiny pieces $1 \mathrm{~mm}^{3}$. The minced pieces were collected in sterile, centrifuge tubes and collagenase type I was added for half an hour at room temperature. Cells were sub-cultured when $70 \%$ confluence was reached. The basal culture media consisted of Dulbecco modified Eagle medium (DMEM) (Gibco, Invitrogen Life Technologies, USA), penicillin/streptomycin $100 \mathrm{unit} / \mathrm{mL}$ and Fungizone, $0.25 \mu \mathrm{g} / \mathrm{mL}$. DPSCs were directly cultured in SF/XF culture medium which constituted the basal culture medium supplemented by $1 \%$ insulin transferrin selenium (ITS), $25 \mu \mathrm{g} / \mathrm{mL}$ ascorbic acid, $25 \mathrm{ng} / \mathrm{mL}$ basic fibroblast growth factor (bFGF) (Sigma-Aldrich, UK), $50 \mu \mathrm{g} / \mathrm{mole}$ $\beta$-mercaptoethanol (Sigma-Aldrich, UK) and $30 \mu \mathrm{g} / \mathrm{mL}$ cholesterol. For control, DPSCs were grown in basal media enriched by $10 \%$ FBS. Cells of the third passage from six different donors were utilised in all experiments.

\section{Flow cytometric surface marker expression analysis for identification and characterization of isolated DPSCs}

Flow cytometric analysis (FACS) for CD90, CD105 and CD34 was performed to confirm the presence of MSCs in the isolated cells and also to rule out the existence of any non-MSCs. Cytomics FC500 flow cytometer (Beckman coulter, USA) and CXP software version 2.2 was used. The expression of MSC markers was quantified according to the following steps: adherent cells were detached and adjusted to $1 \times 10^{6}$ cells $/ \mathrm{mL}$. Then $1 \times 10^{6}$ cells were incubated with $10 \mathrm{~mL}$ of monoclonal antibodies against: CD34 to illustrate hematopoietic negativity in addition CD90 and CD105 to illustrate mesenchymal positivity (Beckman coulter, USA) at $4{ }^{\circ} \mathrm{C}$ in the dark. Isotypes served as negative control. Following incubation for $20 \mathrm{~min}, 2 \mathrm{~mL}$ of PBS supplemented with $2 \%$ FBS was administered to tubes of monoclonal treated cells. Centrifugation for $5 \mathrm{~min}$ at 2,500 rpm was then performed followed by discarding of the supernatant and re-suspending of cells in $500 \mathrm{~mL}$ PBS with $2 \%$ FBS.

\section{Assessment of cell viability}

\section{Cell count and viability}

Stem cells are often considered challenging to counting since they present different morphological appearance. To determine the number of viable and non-viable cells Countess II FL Automated Cell Counter (ThermoFisher Scientific, MA, USA) was used. The analysis parameters of the Countess cell counter were adjusted to compensate for these characteristics, so that stem cells can be easily and accurately counted (7). 
Flow cytometric surface marker expression analysis of apoptotic marker annexin $\mathbf{V}$

For detection of apoptosis, the annexin $\mathrm{V}$ binding capacity of treated cells was examined by flow cytometry using annexin V FITC Detection Kit (BD Pharmingen, San Jose, CA, USA) following the manufacturer's protocol. Since externalization of phosphatidylserine occurs in the early stages of apoptosis, annexin $\mathrm{V}$ fluorescein isothiocyanate (FITC) staining was used in order to determine apoptosis at an earlier stage than tests relying on nuclear alterations like DNA fragmentation. At sub-confluence, DPSCs were incubated at room temperature in $5 \% \mathrm{CO}_{2}$ incubator for 1 day. Cells were washed for 2 times with chilled PBS and re-suspended in $1 \times$ binding buffer at concentration $1 \times 10^{6} / \mathrm{mL}$. Annexin FITC and propidium iodide (PI) were supplemented to $100 \mu \mathrm{L}$ of cell suspension and incubated for $10 \mathrm{~min}$ in the dark. Following incubation $400 \mu \mathrm{L}$ of $1 \times$ binding buffer was added. Stained cells were assessed within an hour by FACS (8).

\section{Assessment of cell proliferation via MTT assay}

Control and treated cells were incubated with MTT [3(-4,5-dimethylthiazol-2-yl)2,5-diphenyltetrazolium bromide] $(0.2 \mathrm{mg} / \mathrm{mL})$ diluted in the appropriate media, for $60 \mathrm{~min}$ at room temperature in 96-well plates. Culture media was discarded and formazan was solubilized. The extent of reduction of MTT to formazan within cells was analysed by a spectrophotometer at a wavelength of $572 \mathrm{~nm}$. Absorbance is indicative of the number of living cells in culture. Cellular proliferation was measured on days 1, 7 and 14 according the cell proliferation kit protocol (Vybrant, Invitrogen). The experiment was repeated in triplicates. This protocol was previously optimized for DPSCs (9).

\section{In vitro multilineage differentiation analysis}

DPSCs cultured in the proposed media were assessed for their multilineage differentiation capacity by lineage specific staining. Differentiation was initiated at a density of 5,000 cells/well in 12 well plates. Following cellular attachment, the culture medium was replaced by adipogenic and chondrogenic differentiation medium. Cells were cultured for three weeks prior to differentiation assessment. DPSCs were stained with $0.3 \%$ Oil Red O solution to identify the presence of extracellular lipid droplets. Fixation with $4 \%$ formaldehyde for $60 \mathrm{~min}$ was performed followed by distilled water wash and incubated in $60 \%$ isopropanol for $5 \mathrm{~min}$. After that, Oil red $\mathrm{O}$ solution was used to stain the cells for $15 \mathrm{~min}$ at $37^{\circ} \mathrm{C}$. As for chondrogenic differentiation, Alcian blue stain was used to detect sulphated glucose amino glycan. Briefly, cells were rinsed with $0.5 \mathrm{~N} \mathrm{HCl}$, then stained with $0.25 \%$ Alcian blue $8 \mathrm{GX}$ (Sigma) in $0.5 \mathrm{~N} \mathrm{HCl}$ overnight. Excess stain was washed off followed by microscopic examination.

\section{Assessment of stemness properties by real-time quantitative PCR}

To assess the effect of the proposed supplemented culture medium on the stem cell properties of the cultured DPSCs, gene expression analysis for markers of stemness (OCT4, $S O X$ and $N A N O G$ ) was carried out. The expression of these markers indicates both the characteristic selfrenewal capacity and the undifferentiated state of stem cells (10). Real-time quantitative polymerase chain reaction analysis differential expressions of the three stemness markers was carried out using the following primers; OCT-4, 5' -AACGACCATCTGCCGCTTTGA-3' and 5'-CTCTCACTCGGTTCTCGATAC-3'; SOX2, 5'-ATCGAGCAGCTGACTACACTG-3' and 5'-TGCGAGTAGGACATGCTGTAG-3'; NANOG, 5'-GAAGGAAGAGGAGAGACAGT-3'. Samples were run twice. Data was analysed with the relative expression software tool (REST) using the automatic cycle threshold (Ct) setting. The relative expressions (REs) of the sample genes were calculated using the $\Delta \Delta C T$ method and GAPDH was used as the internal control. qRT-PCR experiments were carried out in triplicates. Data were presented as the average values \pm standard error of the mean.

\section{Statistical analysis}

Data were reported as mean \pm standard deviation (SD). SPSS 18 (Statistical Package for Scientific Studies) for Windows was implemented for the analysis of data. Statistical significance was analyzed with paired Student $t$-test. Results were considered significant at $\mathrm{P} \leq 0.05$.

\section{Results (Figures 1-4)}

\section{DPSCs cultured successfully in SF/XF culture medium}

DPSCs were successfully isolated enzymatically. Following the isolation and culturing procedures, the DPSCS cultured 
A
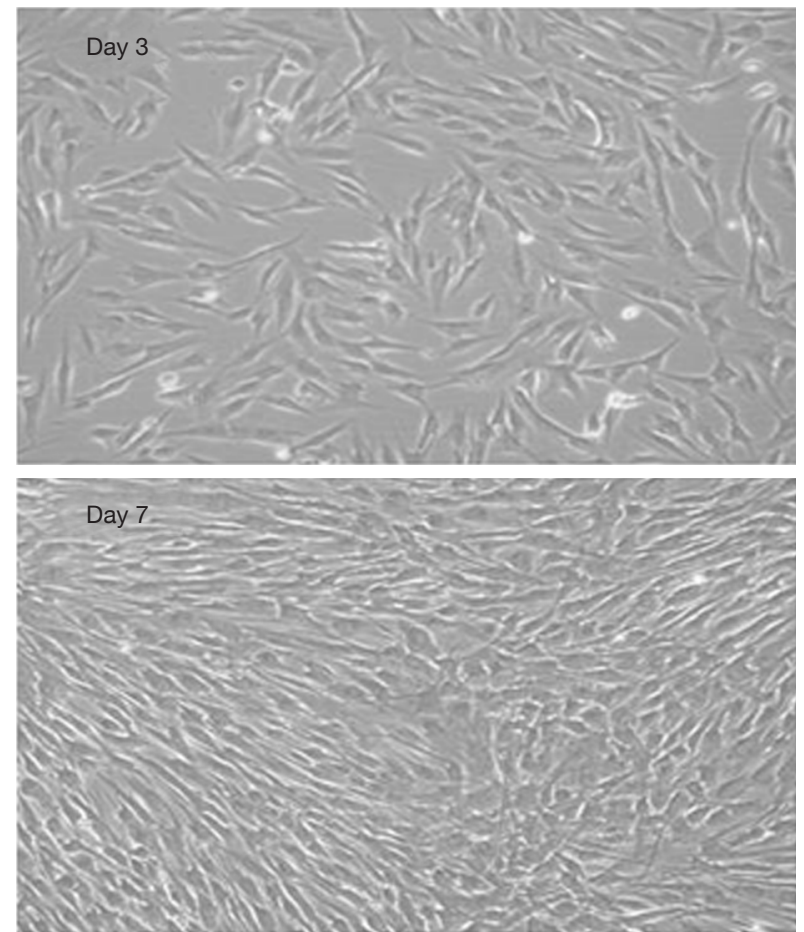
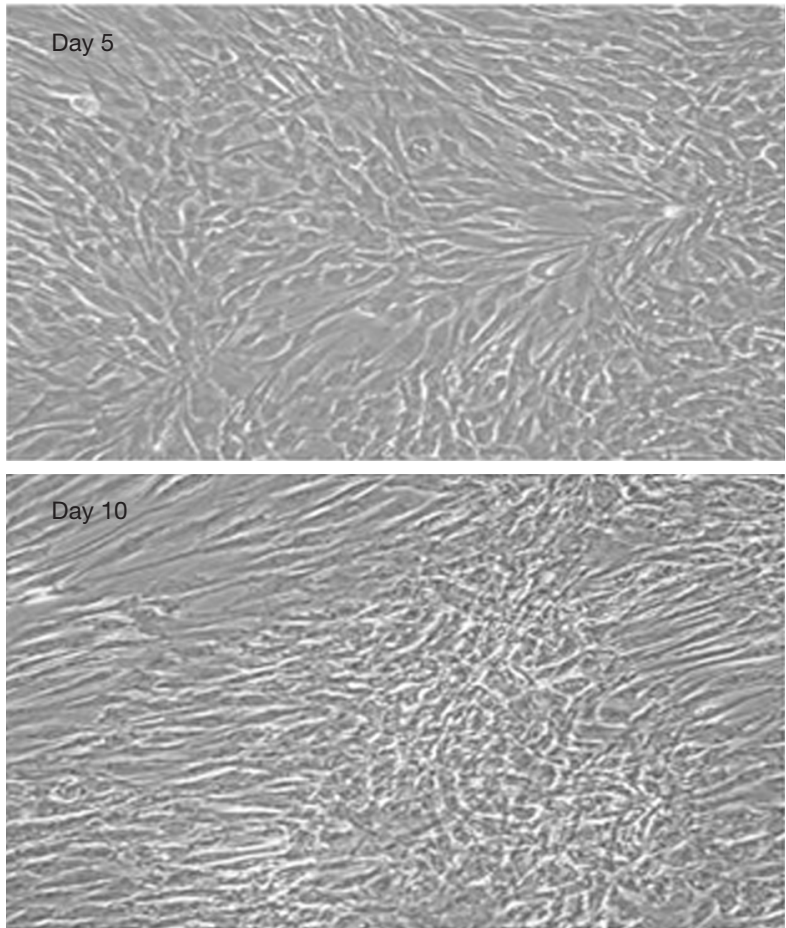

B
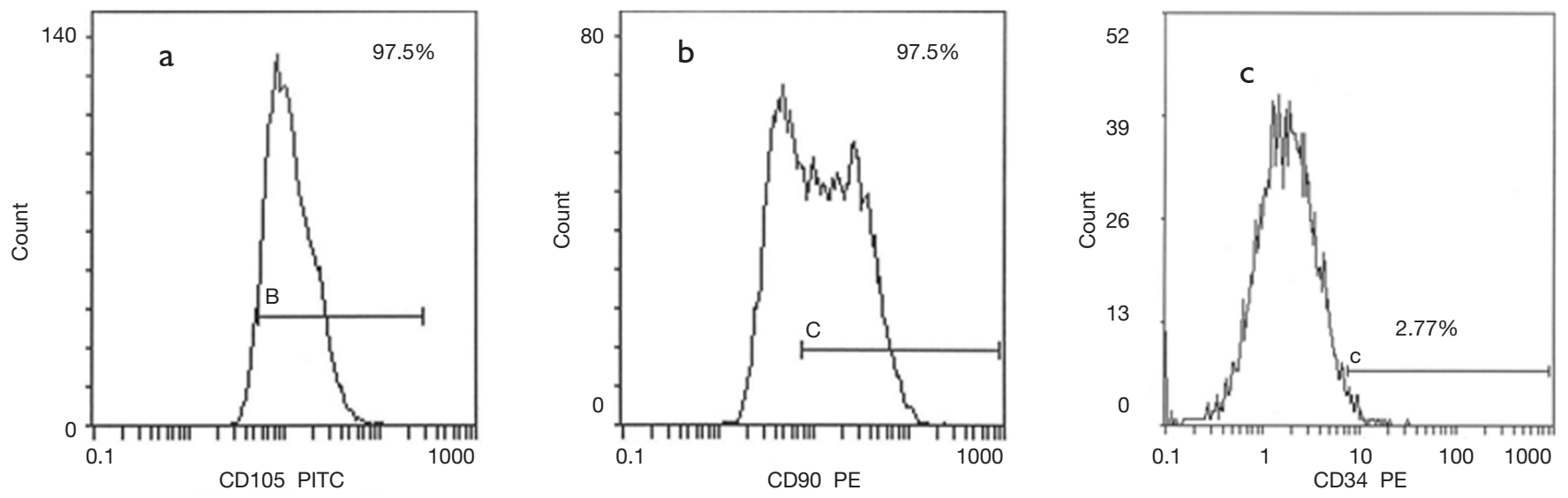

Figure 1 DPSCs cultured successfully in control group. (A) Photomicrograph illustrating morphological appearance of DPSCs cultured in control group. On day 3, spindle-shaped cells started to appear forming a colony. Cells started to increase in number by days $5 \& 7$ while assuming various morphological appearances and reaching confluence by day 10 (scale bar $100 \mu \mathrm{m}$ ). (B) Representative plots of flowcytometry for CD90 and CD105 in DPSCs cultured in basal culture medium supplemented with serum, showing positive expression mesenchymal cell surface markers, and negative expression of hematopoietic cell surface markers CD34. DPSCs, dental pulp stem cells.

in the proposed medium started to adhere to the culture dishes. Cell cultures were observed on the regular basis using an inverted light microscope. The cultured cells in the proposed medium formulation presented normal spindle- shaped fibroblast like morphology, as observed by inverted light microscopy which was similar to the control group (Figure $1 A$ ) though a more homogenous appearance was presented by cells in the proposed medium (Figure $2 A$ ). 
A
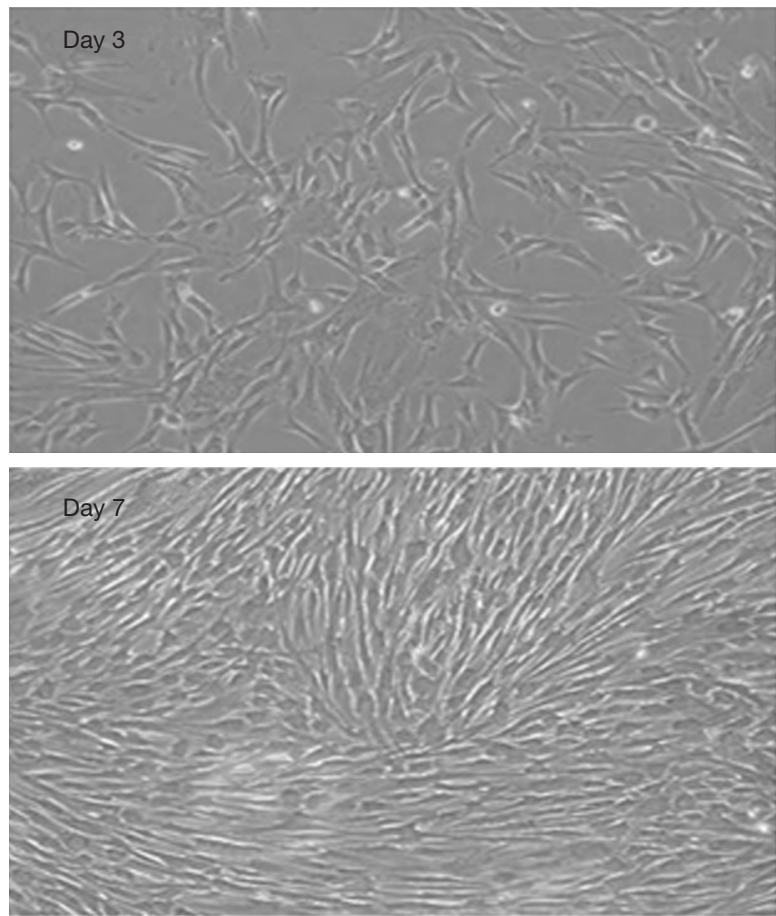
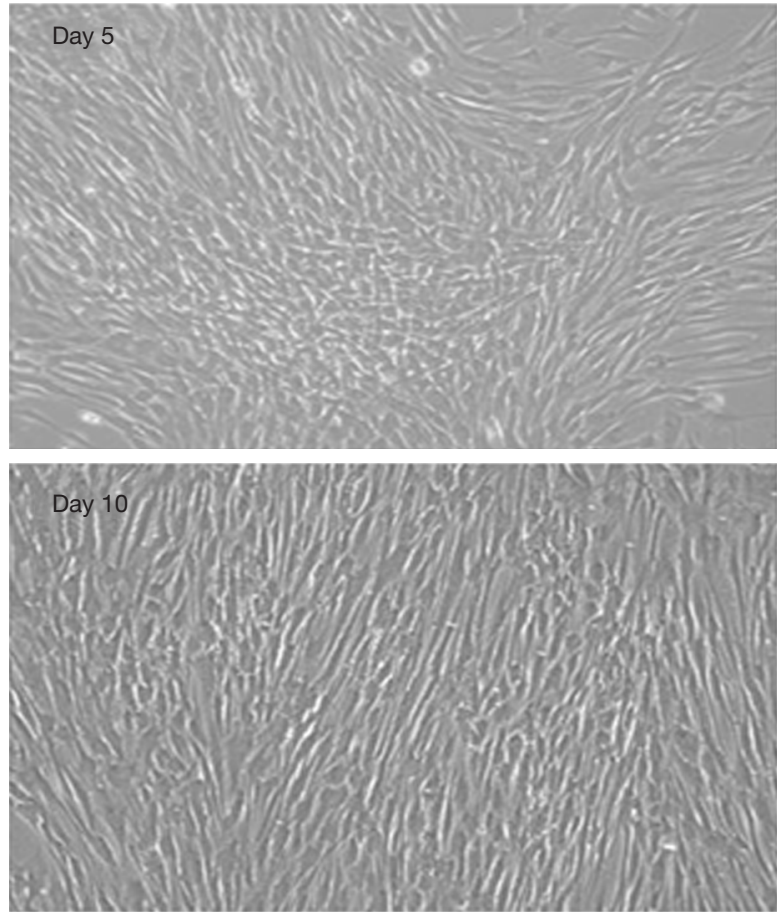

B
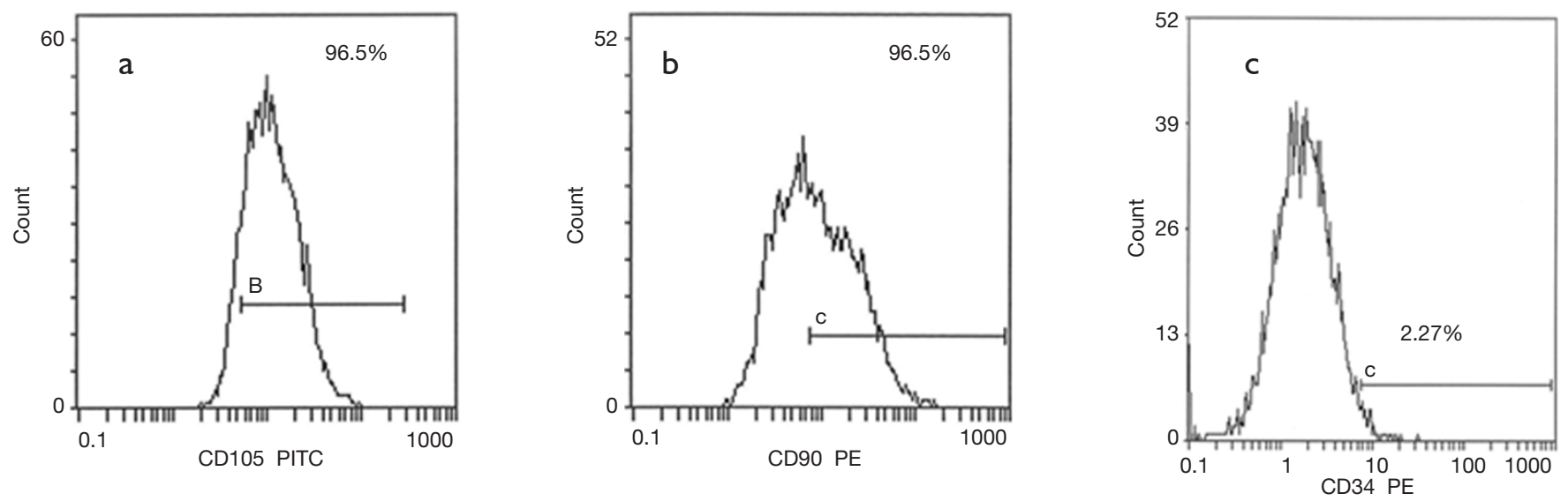

Figure 2 DPSCs cultured successfully in serum-free/xeno-free (SF/XF) culture medium. (A) Photomicrograph illustrating morphological appearance of DPSCs cultured in SF/XF medium on days 3, 5 and 7. Similar morphological appearances to control group was illustrated (scale bar $100 \mu \mathrm{m}$ ). (B) Representative plots of flow-cytometry for CD90 and CD105 in DPSCs cultured in SF/XF medium, showing positive expression mesenchymal cell surface markers, and negative expression of hematopoietic cell surface markers CD34. DPSCs, dental pulp stem cells.

\section{DPSCs cultured in SF/XF culture medium express MSC surface markers}

Furthermore, the expression profile of typical positive and negative hMSCs markers for DPSCs cultured in the proposed culture medium and the expression of CD105, CD90 and CD34 was assessed. FACS analysis demonstrated that DPSCs cultured in the SF/XF culture medium illustrated positive expression for the mesenchymal markers (over 90\%) and negative expression for hematopoietic marker CD34 confirming MSCs characteristics (Figure 2B) which was similar to the results obtained from the control group (Figure 1B). 

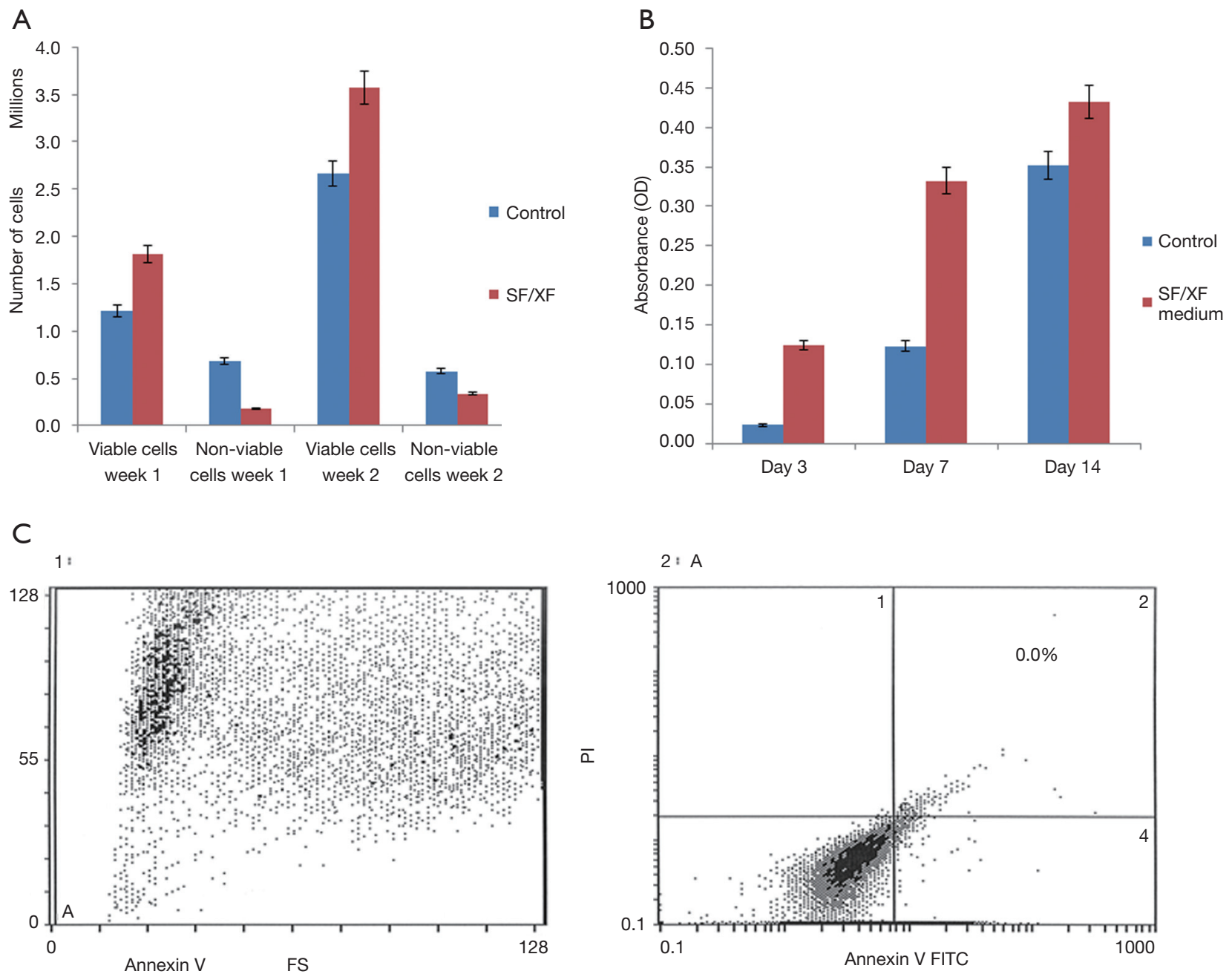

Figure 3 DPSCs cultured in serum-free/xeno-free (SF/XF) culture medium demonstrate enhanced cell viability and proliferation capacity. (A) Cell count of cultured DPSCs after the first and second week of culture. (B) MTT assay of DPSCs on days 3, 7 and 14. Proliferation of DPSCs was significantly higher on days 7 and 14. Statistical significance was analysed with paired Student t-test. Results are illustrated as mean \pm SEM of triple independent experiments and were expressed in $\mathrm{P}$ values. Results were considered significant at $\mathrm{P} \leq 0.05$. (C) Representative plots of flow-cytometry for apoptotic cell surface markers annexin V in DPSCs cultured in SF/XF medium, showing negative expression. DPSCs, dental pulp stem cells; SEM, standard error of the mean.

\section{DPSCs cultured in SF/XF culture medium demonstrate enhanced cell viability and proliferation capacity}

Viable and non-viable cells were both counted to determine cell viability where a marked increase of viability in cells cultured in SF/XF medium was demonstrated. Moreover, minimal expression of apoptotic marker Annexin $\mathrm{V}$ was detected via flow cytometry indicating that the proposed SF/ XF culture media implemented did not affect the survival of the cells. Furthermore, the effect of SF/XF medium on DPSCs growth was assessed on after 1 day, 1 week and 2 weeks via MTT. DPSCs maintained in SF/XF medium proliferated faster than the control group containing FBS as illustrated from day 7. Statistical analysis affirmed that cells maintained in SF/XF medium proliferated significantly faster than cells cultured in control group on days 7 to 14 (Figure 3). 

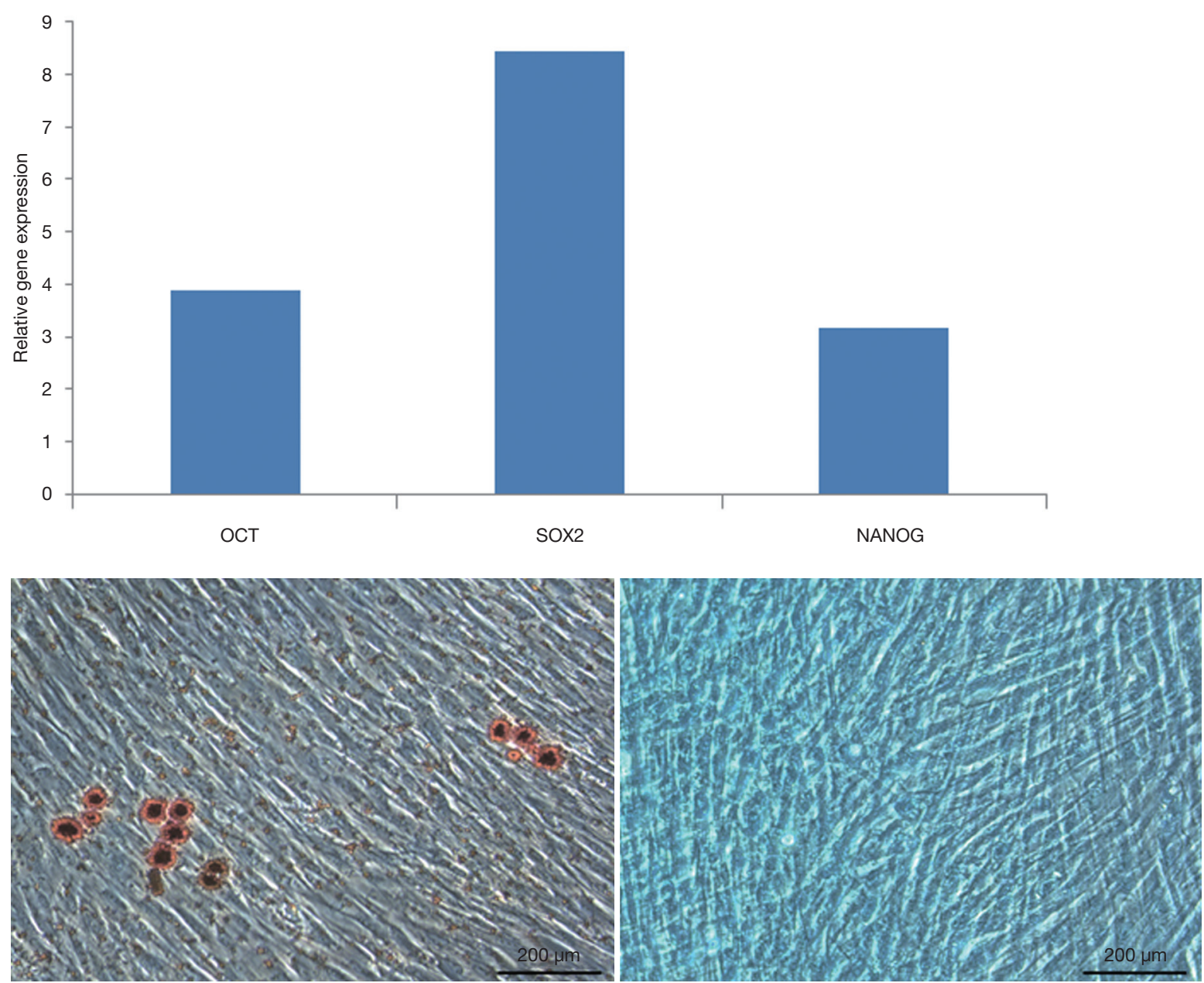

Figure 4 DPSCs cultured in serum-free/xeno-free (SF/XF) culture medium demonstrate stemness characteristics. (A) Quantitative RT-PCR for mRNA levels of stemness specific genes; OCT4, SOX and NANOG, showing up-regulation of those genes in DPSCs grown in SF/XF medium. Results are presented in fold change in relation to control. Results are presented as mean \pm SEM of three independent experiments $(\mathrm{n}=3) .{ }^{*}, \mathrm{P}<0.05$. (B) Adipogenic differentiation of DPSCs was evaluated by Oil Red O staining (right) and revealed accumulated lipid droplets (red) in differentiated cells. Chondrogenic differentiation ability of DPSCs in monolayers was evaluated by Alcian blue staining (left) (scale bar $200 \mu \mathrm{m})$.

\section{DPSCs cultured in SF/XF culture medium express stemness markers}

Gene expression analysis of DPSCs cultured in SF/XF culture medium, revealed a significant increase in expression of stemness markers OCT4, SOX and NANOG in relation to cells maintained in serum-free culture medium. This combination of gene expression depicts the stemness potential of DPSCs cultured in the proposed medium (Figure 4A).

\section{DPSCs cultured in SF/XF culture medium successfully differentiate into adipogenic and chondrogenic lineage}

DPSCs cultured in the proposed SF/XF medium were stimulated to differentiate into adipogenic and chondrogenic lineage for 21 days. Accumulated lipid droplets were positively stained by Oil Red stain indicating successful adipogenic differentiation of the cultured cells. Similarly, Alcian blue stain, illustrated the presence of proteoglycan rich extracellular matrix denoting successful 
chondrogenic differentiation (Figure 4B).

\section{Discussion}

Regenerative medicine is an interdisciplinary approach that seeks to replace diseased cells and tissues in an attempt to re-establish normal function; it involves transplantation of stem cells which could be isolated from different tissues. Among these cells are the DPSCs. It is currently believed that DPSCs shall be pivotal in medical therapy in the future, due to their ability to differentiate into different types of cells and its ability be cryopreserved for long periods (6,11-13). Stem cells are routinely maintained in media supplemented by animal derived components as FBS which is an important crucial adjuvant necessary for cellular proliferation since it contains a large number of extracellular matrix molecules and growth factors useful in cell attachment proliferation and differentiation $(5,14)$. However, disadvantages due to ethical and scientific reasons render FBS a less desirable component of cell culture media (15). Thus, there is a growing demand for optimizing a well-defined culture media that could safely increase the efficacy and reproducibility of the cultured cells especially when clinical applications are intended. In the present study, a cocktail of various essential supplements was carefully selected in an attempt to optimize a SF/ $\mathrm{XF}$ culture medium that is efficient in culturing and maintaining human DPSCs. This cocktail consisted of recombinant human bFGF (hbFGF), ITS, Ascorbic acid, $\beta$-mercaptoethanol and cholesterol. Recombinant hbFGF is one of the growth factors known to enhance stem cell proliferation. It also plays a role in stimulation of selfrenewal capacity of endogenous somatic stem cells within organs, and is committed to the inhibition of senescence in stem cells (16-18). As for ITS, it was selected since it contains; insulin which promotes glucose and amino acid uptake by the cell and the synthesis of proteins and nucleic acids, in addition to transferrin an iron carrier that allows reduction of toxic levels of oxygen radicals and peroxide and finally selenium which is one of the trace elements used as an anti-oxidant in media (19). Another antioxidant that was implemented was ascorbic acid (vitamin C) which was reported to enhance cell growth and proliferation and also aids in the formation of extracellular matrix (collagen, glycosaminoglycan) (20). $\beta$-mercaptoethanol was also added to maintain a reducing environment for the cells in order to protect them from toxic oxygen radicals (20). Finally, there was a need for a member of the lipids family in this cocktail to serve as an energy store during culturing. Cholesterol was selected since it is a naturally occurring structural constituent of the cellular membrane, and plays a role in transport and signalling systems (18). It was previously reported that MSCs cultured in serum-free media from the initial stages including isolation, outperformed MSCs in serum-supplemented media regarding cell growth, viability at isolation and potential for bone repair and showed a comparable biological stability to cells cultured in substitute serum medium (SSM) $(21,22)$. Hence, we adapted DPSCs from the beginning to SF/XF culture medium which was in contrary to previous studies that described a process of gradual weaning involving the progressive adaptation of the cultured cells to reduce serum concentrations up till serumfree conditions are attained (18). However, an unwanted change in the cell population selection might occur, as a sequence of this adaptation process, by indirectly selecting cells capable of growth in serum-free media. After culturing DPSCs in this media, the MSC identity of the isolated cells was characterized by their capability to attach to plastic culture dishes and was confirmed by FACS of stem cell markers; CD90, CD105 and CD34. It is worthy to mention that in our study, during culturing of stem cells in the proposed medium, great care during handling was followed in order to preserve cell viability. Since the beginning of the culturing procedures, several points were followed according to the recommendations of previous studies (23-27). In accordance to previous studies, we started culturing of DPSCs at a rather higher seeding density, also, passaging and sub-culturing of cells were initiated in midlogarithmic phase of growth (28). During the dissociation steps when passaging and sub-culturing procedures were performed, clumping of cells was sometimes reported due to the absence of serum proteins (26). In our study, in order to avoid this clumping, longer incubation time and gentle trituration was followed to break down clumps and to obtain separate single cells. It is important to point out that although there was no use of serum during culturing of stem cells in our study, however we used minimal concentration of FBS during deactivation of trypsin when cells were sub-cultured. In the future, we recommend the use of mechanical detachment to ensure that the cells were not subjected to any source of serum. Another alternative would be the replacement of trypsin by a less harmful protease (e.g., recombinant trypsin) for cell harvesting to avoid the use of FBS. In order to monitor cell viability, DPSCs 
were counted by automated cell counter for easy and more reliable results using trypan blue stain following the first and second weeks. There was no significant difference in the number of isolated cells at day 7; however, the highest number of cells was demonstrated on day 14 . We also analyzed the expression of the early apoptotic marker; annexin $\mathrm{V}$ as an indication of possible apoptosis inflicted by any of the supplements. annexin $\mathrm{V}$ analysis was reported to be more reliable than assays relying on nuclear alterations including DNA fragmentation (29). Its expression by flow cytometry was nil in DPSCs cultured in our proposed media, indicating that the supplements tested did not exhibit any cytotoxic effect. We monitored DPSCs proliferation via MTT assay to further analyse the proliferation pattern of DPSCs cultured in the proposed media. Our data was in agreement with previous studies that showed that bFGF demonstrated a significant proliferative effect on DPSCs (30). This was also in agreement with Sato et al. (22) who demonstrated that the adipose tissue derived-stem cells isolated and cultured in serum-free medium (ATMSCs/SFM) possessed high proliferation capacity. To confirm the proliferative effect achieved by DPSCs cultured in the SF/XF culture medium did not affect the stemness of the isolated cells, we investigated the defining criterion of MSCs to undergo multilineage differentiation. DPSCs cultured in the proposed medium successfully differentiated into adipogenic and chondrogenic lineage. Furthermore, molecular assessment of OCT4, SOX and NANOG expression confirmed the non-altered stemness property of the cultured cells. Recent findings have proposed that intracellular stemness markers OCT4, SOX and NANOG can be used as specific markers to detect DPSCs with high multipotent differentiation potential. Our data revealed significant up-regulation of these genes in DPSCs cultured in our proposed SF/XF culture medium which indicated that the proposed combination of supplements not only did it increase the proliferation potential of DPSCs but, it also enhanced their stemness properties. Taken together, it could be concluded from our results, that the presented culture medium can be considered both safe and efficient in promoting the proliferation capacity of DPSCs. Finally, in an era where there is an increasing demand for safe and ethically accepted alternatives for application in regenerative medicine and cell-based therapies; our proposed culture medium can be considered a valid powerful and promising option that can readily substitute traditional animal derived supplements.

\section{Acknowledgments}

None.

\section{Footnote}

Conflicts of Interest: The authors have no conflicts of interest to declare.

Ethical statement: All experiments conducted in this study have been reviewed and were granted approval by the Ethical Committee of the National Research Centre and Faculty of Dentistry (Ain Shams University) (No. FDASU 503). Informed consents were obtained from donors prior to extraction. The authors are accountable for all aspects of the work in ensuring that questions related to the accuracy or integrity of any part of the work are appropriately investigated and resolved.

\section{References}

1. Potdar PD, Jethmalani YD. Human dental pulp stem cells: Applications in future regenerative medicine. World $\mathrm{J}$ Stem Cells 2015;7:839-51.

2. Huang GT, Gronthos S, Shi S. Mesenchymal stem cells derived from dental tissues vs. those from other sources: their biology and role in regenerative medicine. J Dent Res 2009;88:792-806.

3. Liu J, Yu F, Sun Y, et al. Concise reviews: Characteristics and potential applications of human dental tissue-derived mesenchymal stem cells. Stem Cells 2015;33:627-38.

4. Casagrande L, Cordeiro MM, Nör SA, et al. Dental pulp stem cells in regenerative dentistry. Odontology 2011;99:1-7.

5. Gstraunthaler G, Schöffl H, Appl H, et al. Alternatives to the use of fetal bovine serum (FBS): A survey of recent strategies to reduce or replace FBS in cell and tissue culture. ALTEX 2008;25(Suppl. Linz).

6. Gronthos S, Mankani M, Brahim J, et al. Postnatal human dental pulp stem cells (DPSCs) in vitro and in vivo. Proc Natl Acad Sci U S A 2000;97:13625-30.

7. Ratcliffe E, Glen KE, Workman VL, et al. A novel automated bioreactor for scalable process optimisation of haematopoietic stem cell culture. J Biotechnol 2012;161:387-90.

8. Nedel F, Soki FN, Conde MC, et al. Comparative analysis of two colorimetric assays in dental pulp cell density. Int Endod J 2011;44:59-64. 
9. Guan Z, Shi S, Samruajbenjakun B, et al. Fabrication, characterization and cell cultures on a novel chitosan scaffold. Biomed Mater Eng 2015;25:121-35.

10. Khanna-Jain R, Vanhatupa S, Vuorinen A, et al. Growth and Differentiation of Human Dental Pulp Stem Cells Maintained in Fetal Bovine Serum, Human Serum and Serum-free/Xeno-free Culture Media. J Stem Cell Res Ther 2012;2:126.

11. Ahmed NE, Aly RM, El-Massieh PM, et al. Comparing the Stemness Properties of Dental Pulp Cells (DPSCs), Collected, Processed, and Cryopreserved under Different Conditions. J Stem Cells 2018;13:95-106.

12. Papaccio G, Graziano A, D'Aquino R, et al. Long-term cryopreservation of dental pulp stem cells (SBP-DPSCs) and their differentiated osteoblasts: A cell source for tissue repair. J Cell Physiol 2006;208:319-25.

13. Huynh NC, Le SH, Doan VN, et al. Simplified conditions for storing and cryopreservation of dental pulp stem cells. Arch Oral Biol 2017;84:74-81.

14. Kazemnejad S, Allameh A, Gharehbaghian A, et al. Efficient replacing of fetal bovine serum with human platelet releasate during propagation and differentiation of human bone marrow-derived mesenchymal stem cells to functional hepatocytes-like cells. Vox Sang 2008;95:149-58.

15. Rauch C, Feifel E, Amann EM, et al. Alternatives to the use of fetal bovine serum: human platelet lysates as a serum substitute in cell culture media. ALTEX 2011;28:305-16.

16. Coutu DL, François M, Galipeau J. Inhibition of cellular senescence by developmentally regulated FGF receptors in mesenchymal stem cells. Blood 2011;117:6801-12.

17. Jung J, Moon N, Ahn JY, et al. Mesenchymal stromal cells expanded in human allogenic cord blood serum display higher self-renewal and enhanced osteogenic potential. Stem Cells Dev 2009;18:559-71.

18. van der Valk J, Mellor D, Brands R, et al. The humane collection of fetal bovine serum and possibilities for serumfree cell and tissue culture. Toxicol In Vitro 2004;18:1-12.

19. Freshney RI. Differentiation. In: Culture of Animal Cells. Hoboken, NJ, USA: John Wiley \& Sons, Inc., 2005:291-8. 20. Ishkitiev N, Yaegaki K, Calenic B, et al. Deciduous and

doi: $10.21037 /$ sci.2019.06.05

Cite this article as: Abdel Moniem EM, EL-Batran MM, Halawa AM, Gomaa DH, Eldeen GN, Aly RM. Optimizing a serum-free/xeno-free culture medium for culturing and promoting the proliferation of human dental pulp stem cells. Stem Cell Investig 2019;6:15.
Permanent Dental Pulp Mesenchymal Cells Acquire Hepatic Morphologic and Functional Features In Vitro. J Endod 2010;36:469-74.

21. Chen G, Yue A, Ruan Z, et al. Human umbilical cordderived mesenchymal stem cells do not undergo malignant transformation during long-term culturing in serum-free medium. PLoS One 2014;9:e98565.

22. Sato K, Itoh T, Kato T, et al. Serum-free isolation and culture system to enhance the proliferation and bone regeneration of adipose tissue-derived mesenchymal stem cells. In Vitro Cell Dev Biol Anim 2015;51:515-29.

23. van der Valk J, Brunner D, De Smet K, et al. Optimization of chemically defined cell culture media--replacing fetal bovine serum in mammalian in vitro methods. Toxicol In Vitro 2010;24:1053-63.

24. Brunner D, Frank J, Appl H, et al. Serum-free cell culture: the serum-free media interactive online database. ALTEX 2010;27:53-62.

25. Chase LG, Lakshmipathy U, Solchaga LA, et al. A novel serum-free medium for the expansion of human mesenchymal stem cells. Stem Cell Res Ther 2010;1:8.

26. Jung S, Panchalingam KM, Rosenberg L, et al. Ex vivo expansion of human mesenchymal stem cells in defined serum-free media. Stem Cells Int 2012;2012:123030.

27. Zheng X, Baker H, Hancock WS, et al. Proteomic analysis for the assessment of different lots of fetal bovine serum as a raw material for cell culture. Part IV. Application of proteomics to the manufacture of biological drugs. Biotechnol Prog 2006;22:1294-300.

28. Jung JY, Woo SM, Lee BN, et al. Effect of Biodentine and Bioaggregate on odontoblastic differentiation via mitogenactivated protein kinase pathway in human dental pulp cells. Int Endod J 2015;48:177-84.

29. Kanjevac T, Milovanovic M, Volarevic V, et al. Cytotoxic effects of glass ionomer cements on human dental pulp stem cells correlate with fluoride release. Med Chem 2012;8:40-5.

30. Wu J, Huang GT, He W, et al. Basic fibroblast growth factor enhances stemness of human stem cells from the apical papilla. J Endod 2012;38:614-22. 